\title{
Breves consideraciones sobre el sistema colonial en Puerto Rico
}

\author{
Breves considerações sobre o sistema colonial em Porto Rico \\ Brief observations on the colonial system in Puerto Rico
}

Alejandro Schneider

\section{Resumen}

A partir de la firma del Tratado de París de 1898, Puerto Rico se convirtió en una colonia estadounidense. Desde ese momento hasta el presente, la metrópoli norteamericana ha empleado diversos instrumentos de control para ejercer su presencia en el archipiélago. El propósito de este artículo es analizar algunos rasgos que manifiesta esta dominación imperial. En particular, las observaciones se detienen a examinar los mecanismos usados por los Estados Unidos para asimilar a los puertorriqueños sin integrarlos políticamente a su país. A la vez, se considera cómo estas herramientas también se utilizan para impedir toda expresión a favor de la independencia.

Palabras clave: Colonia dominación independência.

\section{“No quiero la colonia con España ni con Estados Unidos. \\ ¿Qué hacen los puertorriqueños que no se rebelan?" \\ Ramón Emeterio Betances}

Este interrogante formulado hace más de cien años por uno de los máximos líderes independentistas puertorriqueños ha provocado, hasta el día de hoy, una intensa y variada gama de reflexiones; numerosas páginas han sido escritas sobre estas observaciones. En consonancia con esta preocupación, y lejos de dar una mirada acabada sobre el tema, este artículo se propone esbozar algunos rasgos que presenta la dominación estadounidense en Puerto Rico, la colonia más antigua del mundo.

Doctor en Historia. Universidad Nacional de La
Plata. Universidad de Buenos Aires.

Recebido em: 29/08/2012 Aprovado em: 28/11/2012

http://dx.doi.org/10.5335/hdtv.13n.1.2593 
El archipiélago de Puerto Rico fue descubierto por los españoles durante el segundo viaje de Cristóbal Colón en 1493. Años más tarde, a partir de 1506, el territorio comenzó a ser explotado por los conquistadores ibéricos, primero extrayendo oro y luego, diferentes productos agrícolas: azúcar, café y tabaco. Sin embargo, esta injerencia colonial no fue totalmente sencilla. En el transcurso de su historia hubo una miríada de revueltas populares que cuestionaron el dominio europeo. Desde las primeras rebeliones guiadas por el cacique Agueynaba el Bravo en 1511, pasando por la sublevación de "los vecinos" de 1701-1712, hasta el movimiento de 1808 - 1812, distintos sectores de la población boricua buscaron alguna forma de emancipación.

A pesar de los sucesivos fracasos, la idea de independizarse de España permaneció a lo largo del siglo XIX. La máxima expresión de este pensamiento se manifestó en el mes de septiembre de 1868 con el Grito de Lares. Durante esas jornadas se produjo el principal estallido popular con el fin de crear una nación libre y soberana. Organizadas intelectual y militarmente por Ramón Emeterio Betances, cerca de un millar de personas, protagonizaron una insurrección armada declarando la República de Puerto Rico. Si bien la revolución fue rápidamente derrotada por las tropas hispanas, su legado permanece en el tiempo; tal como lo rememoran los diversos sectores partidarios de la liberación colonial. ${ }^{1}$

Al calor de la derrota de Lares fue naciendo con fuerza, en diferentes sectores de la sociedad, el pensamiento reformista o autonomista. Esta corriente ideológica, de manera consecuente a lo largo de la historia y a través de distintas formas, siempre ha buscado acordar alguna clase de pacto con los poderes metropolitanos de turno; conformándose con un limitado número de potestades y de derechos. Primero, los seguidores del autonomismo negociaron con el decadente imperio español en la década de 1870, luego con los Estados Unidos, tras la invasión de 1898. En definitiva, junto con los acérrimos defensores de la incorporación ultramarina, los distintos apologistas del reformismo se opusieron a crear un estado emancipado. Desde entonces, la historia de Puerto Rico se encuentra cruzada por una fuerte y permanente tensión entre los partidarios de la anexión, el autonomismo y los independentistas. Cabe indicar que esta problemática se expresó (y aún se manifiesta) en diferentes planos de la sociedad; ésta traspasa todos los espacios políticos, económicos, sociales, culturales e ideológicos.

A partir de la firma del Tratado de París de 1898, el archipiélago caribeño pasó a depender del Congreso de los Estados Unidos. Poco tiempo después, el dominio colonial se reforzó con la Ley Foraker de 1900 (por la cual se creó un "gobierno civil" digitado desde Washington) y con la Ley Jones de 1917, por la que se impuso a los puertorriqueños la ciudadanía estadounidense. De este modo, junto con diferentes resoluciones del Tribunal Supremo norteamericano, se estableció la condición de territorio no incorporado; en otras palabras, Puerto Rico pertenece a pero no forma parte de los Estados Unidos. Asimismo, se acordó que seguía siendo tan sólo una posesión territorial, no existiendo la intención de incorporarlo en el futuro 
como parte de la unión. En la práctica, se convirtió en una gran plantación azucarera, con gobernadores yanquis designados por el presidente de turno, con la bandera del Tío Sam como única enseña, con el intento (infructuoso) de establecer el idioma inglés en la población y con la radicación de numerosas bases militares. ${ }^{2}$

Desde sus inicios, Puerto Rico resultó ser un importante enclave geopolítico para los Estados Unidos en el mar Caribe. Esto se expresó en que las islas fueron sede de numerosas operaciones militares en el área. Ha servido como cabecera de playa para varias invasiones en la región. Además, los jóvenes puertorriqueños se han visto obligados al servicio militar y a combatir en diferentes regiones del mundo. En idéntico sentido, ha desempeñado un papel de vidriera simbólica de un modelo supuestamente exitoso de democracia capitalista ante otros países de la zona; en particular, frente a Cuba tras la revolución de 1959.

Por otra parte, sus pobladores se han visto sometidos a distintos tipos de estudios y experimentos medicinales. Entre otros, uno de los casos más renombrados, y reconocido por el propio Estados Unidos, fue un ensayo humano que se hizo, entre 1949 y 1951, para conocer los defectos de la vacuna contra la tuberculosis (Bacilo Calmette Guerin BCG) en el que se arriesgó la vida de miles de infantes puertorriqueños.

Ahora bien, ¿cómo se ha sostenido y reproducido esta situación colonial? En el transcurso de su dominio, Estados Unidos implementó diversos mecanismos para mantener el control del archipiélago. En todas las ocasiones se buscó asimilar a los puertorriqueños sin integrarlos políticamente a la metrópoli; a la vez, intentó impedir por cualquier medio la independencia. Para consolidar esta política de intervención, el poder imperial combinó (en distintas coyunturas históricas) instrumentos de orden jurídico, ideológico, económico y represivo.

En términos jurídicos, una de las disposiciones adoptadas fue la de concederles la ciudadanía estadounidense. Su propósito no fue el de incorporarlos como otro estado de su federación; por el contrario, su intención fue frenar el avance de las ideas nacionalistas. En forma simultánea, la Corte Suprema norteamericana validó (en reiteradas ocasiones) la doctrina del "territorio no incorporado", es decir, un territorio que le pertenece y que está bajo su plena soberanía, pero que no forma parte del país. Asimismo, como lo establece el Tratado de París, el Congreso de Washington dicta las principales normas que rigen en la isla; entre otras, la Ley 600 por la que se autoriza al "pueblo" de Puerto Rico la creación de la Constitución del Estado Libre Asociado (ELA).

En términos de conformar un sistema de cooptación ideológica se buscó fomentar la imaginaria creencia de que la sociedad puertorriqueña necesita estar bajo la dependencia norteamericana. Al igual que en otras situaciones coloniales, históricas y contemporáneas, se insistió en la condición de inferioridad a la vez que se realzó los valores supremos de la metrópoli. Esto se hizo por medio de distintos instrumentos: desde el otorgamiento de la ciudadanía, la aprobación de ciertos derechos sociales y civiles, los intentos de sustituir el idioma español o la creación de un nuevo ordenamiento jurídico 
político con la sanción del Estado Libre Asociado, entre otras acciones.

Desde los primeros años del siglo XX, Estados Unidos fue concediendo una serie de concesiones democráticas con el fin de acallar las pretensiones a favor de la independencia. Con la imposición de la ciudadanía, si bien limitada a ciertas prerrogativas legales, se abrió la posibilidad de radicarse en Norteamérica sin los riesgos de permanecer en una situación de ilegalidad. Por otro lado, en el ámbito civil y laboral se otorgaron todo un conjunto de derechos que tendieron a equiparar la situación de la clase trabajadora entre ambos países, evitando que las demandas obreras boricuas empalmen con reclamos emancipadores.

A este panorama se le agregó el control en el ámbito educativo, en particular, el intento de imponer el uso del inglés en el sistema público de enseñanza. Su empleo fue considerado como un requisito necesario para la adquisición de la cultura anglosajona a la vez que se buscó eliminar todo ingrediente que pudiese fortalecer la identidad caribeña. En idéntico sentido, se prohibió el izamiento de la bandera puertorriqueña o la entonación de canciones que aludieran a la emancipación. El discurso colonialista negó, por esos años, sistemáticamente la existencia de una nacionalidad boricua por ende sostuvieron que el pueblo isleño era incapaz de autogobernarse. Sin embargo, tras sucesivas protestas y el crecimiento del Partido Nacionalista, el gobierno estadounidense, en el marco de la guerra fría de la década de 1950, decidió modificar algunas de estas posturas.

Desde entonces, determinados valores que reafirman la puertorriqueñidad fueron aceptados como partes integrantes del aparato ideológico de dominación. De este modo, al incorporarse el español como lengua oficial y la bandera boricua en las oficinas estatales, entre otras prácticas identitarias, se fue institucionalizando y canalizando desde Washington ciertos reclamos del independentismo mientras no se alteraba en lo substancial el proceso colonizador.

Dentro de este escenario, corresponde indicar que el proceso más importante de asimilación fue el establecimiento del Estado Libre Asociado en 1952; por medio del cual, se concedió un estatus político de pseudo soberanía, encubriendo con este manto jurídico una relación de dominación vigente hasta el presente. En el Preámbulo de su Carta Magna se dispone que uno "de los factores determinantes" de la misma es "la ciudadanía de los Estados Unidos de América". Asimismo, se declara subordinada a "los postulados de la Constitución Federal" de dicho país. ${ }^{3}$ En otras palabras, Puerto Rico se transformó en una colonia moderna con acceso a ciertos derechos civiles y sociales, con poderes limitados sobre cuestiones locales atinentes a la educación, vivienda, salud, impuestos y cultura. Por su parte, la metrópoli continuó interviniendo en los asuntos referentes a defensa, moneda, ciudadanía, inmigración, comunicación, transporte, aduana y comercio exterior. Más aún, las leyes norteamericanas pueden anular cualquiera de las disposiciones legales dictadas por el parlamento o las autoridades del archipiélago.

Cabe subrayar que este proceso jurídico fue acompañado, desde entonces hasta el presente, por el accionar de las dos princi- 
pales organizaciones políticas: el Partido Popular Democrático (PPD) y el Partido Nuevo Progresista (PNP). Ambas fuerzas, más allá de ciertas declaraciones de algunos de sus miembros, siempre actuaron en defensa del régimen colonial con Estados Unidos.

La definición institucional de Puerto Rico ha creado una situación que se presta a la confusión y a la ambigüedad dentro de la propia sociedad. Por ejemplo, para un porcentaje significativo de la población coexiste la quimera de los "beneficios" que ofrece el poseer un pasaporte norteamericano con el rescate por las costumbres y las tradiciones isleñas. En términos ideológicos, esta condición administrativa ha permitido que crezca una identidad cultural puertorriqueña que acepta ciertas ventajas del dominio colonial estadounidense. Ahora bien, corresponde subrayar que esta singularidad ha perjudicado la identidad política boricua, debilitando los principios e ideales nacionalistas.

De esta manera, el régimen metropolitano se acomodó para sus propios fines de ciertos componentes culturales que intervienen como dispositivos de control. Diversos emblemas e insignias como la lengua hispana, la tradición de los Reyes Magos, la imagen del jíbaro y su familia, entre otros rituales y símbolos de la memoria colectiva, han sido aceptados como instrumentos de dominación. En la actualidad, desde las oficinas de la gobernación hasta el último despacho oficial conviven en forma simultánea dos himnos, dos banderas y dos idiomas. En forma paralela, a diferencia de lo dispuesto a principios del siglo $X X$, las prácticas de consumo y las costumbres anglosajonas (como el día de Acción de Gracias) se promueven y fomentan en español, a la vez que se nutren de rasgos propios de la identidad puertorriqueña.

En cuanto a los instrumentos económicos, el archipiélago ha pasado por distintos mecanismos de dependencia con la metrópoli. En primer lugar, continuó con la producción de materias primas para su exportación; posteriormente, con el programa conocido como "Operación manos a la obra" se inició un proceso manufacturero según las necesidades del capital yanqui, sobre la base de exiguos salarios y con notables exenciones contributivas para las corporaciones estadounidenses. Todo esto en un marco donde la población es una consumidora cautiva de bienes norteamericanos cuyo valor se encuentra encarecido por el monopolio que ejerce Estados Unidos en el transporte marítimo.

Por otra parte, Puerto Rico desde la década de 1930 ha recibido diversos fondos federales de ayuda del gobierno norteamericano con el fin de conservar la dependencia económica y política. Asimismo, estos a su vez sirvieron para mantener ideológicamente subordinados a vastos grupos de la sociedad.

En el escenario de la guerra fría, como insignia simbólica del triunfo del capitalismo en el Caribe, el país recibió una importante transferencia de dinero de Washington en áreas de educación, salud y vivienda. Sin embargo, sus fines no fueron sólo para uso externo. En el plano doméstico, sobre todo desde la crisis de 1973, la asistencia federal (con cupones de alimentos, seguros sociales, becas Pell, etc.) se dirigió a contener a sectores alcanzados por altos niveles de pobreza. 
Como consecuencia, el incremento de las ayudas gubernamentales incidió en el crecimiento de las simpatías a favor del anexionismo con los Estados Unidos; en particular, esta postura cobró mayor aceptación dentro de los grupos materialmente más vulnerables y desprotegidos.

En este esquema, las fuerzas políticas antes mencionados (PPD y PNP), dentro de la lógica del funcionamiento del ELA, son las que administran las relaciones clientelares con la sociedad. Las organizaciones partidarias intervienen tanto como vehículo de distribución y reparto de las transferencias federales como en la provisión de empleos o viviendas.

En forma paralela, la política de asimilación se consolida con el permanente flujo migratorio de isleños hacia los Estados Unidos. Desde mediados de la década del treinta, el movimiento poblacional se convirtió en una clásica válvula de escape (incluso alentada por los gobiernos) frente a las reiteradas crisis económicas y a la desocupación. Cabe indicar que los desplazamientos son continuos y que se dan en ambos sentidos; por lo tanto, pocas veces se quiebran los lazos sociales, económicos y culturales con la comunidad de origen. La mayoría de los miembros que integran la diáspora se siguen auto referenciándose como boricuas.

De este modo, Puerto Rico se ha conformado en el transcurso del siglo XX (y durante la presente centuria), en términos demográficos, en una nación dividida entre la isla y el continente. Sin embargo, este fenómeno ha provocado disímiles consecuencias. Entre otras, el hecho de que se pueda obtener un empleo gracias a la posesión de la ciudadanía estadounidense ha intervenido en la conveniencia (o no) de luchar por la independencia.

Por último, no por eso menos importante, otros de los factores que operaron para la sujeción política fue la utilización de la represión a través de diferentes aparatos estatales a toda forma de expresión emancipadora o impugnadora de medidas llevadas a cabo por el régimen colonial. La misma se dirigió en forma selectiva tanto a organizaciones como a individuos. Estados Unidos, en función de tales objetivos, ha recurrido a distintos métodos: desde el control de los medios de comunicación hasta el seguimiento de personas, pasando por el uso de detenciones, torturas y asesinatos.

Sin duda, el primer hecho represivo fue la propia ocupación militar del territorio en 1898. Desde ese momento, la vigilancia, el control y el castigo sobre la población fue una práctica coercitiva que se empleó en forma ininterrumpida hasta el presente. Cabe indicar que el accionar estuvo ejercido tanto por diversas agencias federales como por fuerzas de seguridad del gobierno local; en numerosas oportunidades, incluso con la complicidad de agrupaciones políticas, del sistema judicial o la propia delación de los ciudadanos.

El independentismo, en el transcurso de toda su historia, ha sufrido un sinfín de acciones represivas. En las primeras décadas de la ocupación, la coerción se orientó sobre todo a personas provenientes del mundo de la cultura y de los medios de comunicación que cuestionaron la invasión, la enseñanza del inglés y la obligatoriedad del servicio militar. Frente a ello, el régimen colonial 
procesó judicialmente y sentenció a prisión a más de doscientos activistas.

A partir de 1930, con el liderazgo incesante de Pedro Albizu Campos en el Partido Nacionalista, las medidas represivas aumentaron en forma considerable. En primer lugar, la metrópoli volvió a designar como gobernador a un militar: el general Blanton Winship; posteriormente, fortaleció las fuerzas de seguridad locales con el envío de agentes del Buró Federal de Investigaciones (FBI) y con el equipamiento de ametralladoras. El asesinato de cuatro nacionalistas por parte de la policía en Río Piedras (1935) y la masacre efectuada en Ponce (1937) junto con el encarcelamiento de numerosos luchadores como Albizu Campos, entre otros, sintetizaron este cruel escenario de época. Durante la década del cuarenta, la persecución y la represión contaron con el aval de Luis Muñoz Marín y su Partido Popular Democrático, quienes implementaron la Ley de la Mordaza. Bajo esta norma legal las autoridades castigaban a todas aquellas personas o grupos que de manera pública (en forma oral o escrita) defendían los ideales de la independencia. Sin embargo, estas disposiciones no impidieron que prosiguiesen los reclamos por la emancipación. Así lo demostraron la insurrección de octubre de 1950 en varias ciudades del archipiélago, el ataque a la Casa Blair (residencia interina del presidente Harry Truman) y la irrupción armada sobre el Congreso norteamericano en $1954{ }^{4}$

Con el establecimiento del ELA en 1952, las acciones represivas no cesaron; por el contrario, se perfeccionaron. Al calor de las refriegas contra el envío de jóvenes al sudeste asiático, el rechazo a la extracci- ón minera, la campaña por la excarcelación de los presos políticos o las protestas por la bases militares norteamericanas en las islas, los diferentes gobiernos locales junto con los mandatarios estadounidenses desarrollaron un conjunto de medidas persecutorias y represivas. De todas estas disposiciones, una de las acciones más claramente violatorias de los derechos civiles fue la realización de un fichaje institucional, sistemático, permanente y generalizado de independentistas, ambientalistas, sindicalistas, religiosos, feministas, etc. Si bien estos operativos (conocidos con el nombre de "carpeteo") funcionaron desde 1898, alcanzaron su máxima expresión desde la década de los sesenta hasta fines de los ochenta, cuando fueron denunciados en forma pública, aceptando las autoridades su existencia. ${ }^{5}$

Como corolario a estas acciones, y no satisfechos con las mismas, la metrópoli y los mandatarios locales, también asesinaron a más de una docena de nacionalistas: entre otros, a la estudiante Antonia Martínez Lagares (1970) y a los jóvenes Arnaldo Darío Rosado y Juan Soto Arriví en el Cerro Maravilla (1978).

Cabe recalcar que el empleo del accionar represivo también se usó para advertir y atemorizar a la población a través de medidas con un fuerte carácter simbólico. La principal de ellas fue el asesinato del fundador del Ejército Popular Boricua, Filiberto Ojeda Ríos, el 23 de septiembre de 2005, aniversario del Grito de Lares, la jornada más importante para los independentistas puertorriqueños. Al respecto, sobre este crimen, corresponde indicar que el mismo fue perpetrado por agentes de seguridad enviados 
desde Norteamérica debido a que Washington no confiaba, para la realización de esta tarea, en las fuerzas policíacas de la isla. ${ }^{6}$

Finalmente, es necesario subrayar que aún se encuentran en las cárceles federales de Estados Unidos prisioneros políticos independentistas; entre otros, Oscar López Rivera, el convicto más antiguo del hemisferio, con más de treinta años de prisión sobre la base de dos sentencias que en conjunto suman setenta años de condena.

En resumen, el empleo en forma combinada de todos estos instrumentos jurídicos, ideológicos, económicos y represivos, a lo largo de la historia, han colaborado en perpetuar el dominio colonial sobre Puerto Rico. No obstante, la cuestión del "estatus" es un asunto que se discute en todos los ámbitos públicos y privados, desde los medios de comunicación hasta en las escuelas; no hay individuo en el archipiélago que no mencione los términos de estadolibrismo, anexionismo o emancipación. Una irónica expresión de esta problemática, se manifiesta cada 25 de julio. En esa jornada, los primeros celebran la fecha en que se promulgó la Constitución del Estado Libre Asociado (1952); por su parte, los segundos, recuerdan el desembarco estadounidense en las costas de Guánica (1898); mientras que los últimos, rememoran que en ese día se produjeron los asesinatos del Cerro Maravilla (1978).

A pesar de ello, corresponde subrayar que el régimen imperial ha aceptado ciertas ideas y valores que hacen referencia a la propia identidad puertorriqueña; sobre todo, la ha admitido en términos culturales para su beneficio. Sin embargo, cuando esta frontera se la traspasa y se la convierte en una procla- ma política de emancipación se la combate, como ha sido explicado más arriba. De este modo, vale observar que el nacionalismo cultural tiene sus límites y contradicciones; su uso puede ser empleado como herramienta de liberación o de dominación.

El proceso colonizador no le ha resultado sencillo a los Estados Unidos. La historia contra la opresión territorial se encuentra signada de pequeñas y grandes hazañas; desde los ataques nacionalistas concebidos bajo el liderazgo de Albizu Campos hasta el surgimiento de numerosos grupos armados que lucharon por la autodeterminación entre los años sesenta y noventa. En esta permanente impugnación al dominio ultramarino fue que la metrópoli y sus agentes locales buscaron nuevos instrumentos de control. Sin duda, con la creación del ELA se pasó de un método de colonización antiguo a uno moderno; no obstante el cambio de forma, la substancia siguió siendo la misma.

A más de cien años de haberse convertido en un territorio estadounidense, la lucha por la liberación de Puerto Rico transcurre por diversos ámbitos espaciales y por distintos carriles, tanto a nivel nacional como internacional. Pese a ciertos agoreros, la historia no ha llegado a su fin, todavía quedan muchas páginas por escribir y el interrogante de Betances queda aún sin responder.

\section{Resumo}

A partir da assinatura do Tratado de Paris de 1898, Porto Rico se converteu numa colônia estadounidense. Desde esse momento até o presente, a metrópole norte-americana empregou diversos 
instrumentos de controle para exercer sua presença no arquipélago. O propósito deste artigo é analisar alguns rasgos que manifesta essa dominação imperial. Em particular, as observações se detêm a examinar os mecanismos usados pelos Estados Unidos para assimilar aos porto-riquenhos sem integrá-los politicamente a seu país. Ao mesmo tempo, considera-se como essas ferramentas também se utilizam para impedir toda expressão a favor da independência.

Palavras-chave: Colônia dominação independência.

\section{Abstract}

From the signing of the Agreement of Paris of 1898, Puerto Rico became a United States colony. From that time until the present, the North American metropolis has employed various control instruments to exercise its presence in the archipelago. The intention of this article is to analyze some features that manifests this imperial domination. In particular, the comments stop to examine the mechanisms used by the United States to assimilate Puerto Ricans without integrating his country politically. At the same time, it considers how these tools are also used to prevent any expression in favour of the independence.

Keywords: Colony independence domination.
Notas

1 MOSCOSO, Francisco. La Revolución Puertorriqueña de 1868: el Grito de Lares. San Juan: Instituto de Cultura Puertorriqueña, 2003.

2 MALDONADO DENIS, Manuel. Puerto Rico. Una interpretación histórico - social. México: Siglo XXI, 1970.

3 CONSTITUCIÓN DEL ESTADO LIBRE Y ASOCIADO DE PUERTO RICO, 1952.

4 GONZÁLEZ CRUZ, Michael. Nacionalismo revolucionario puertorriqueño. La lucha armada, intelectuales y prisiones políticos y de guerra. San Juan: Isla Negra, 2006.

5 BOSQUE PÉREZ, Ramón; COLÓN MORERA, José. Las Carpetas. Persecución política y derechos civiles en Puerto Rico. Río Piedras: CIPDC, 1997.

6 PARALITICI, José. La represión contra el independentismo puertorriqueño: 1960-2010. San Juan: Ediciones Gaviota, 2011.

\section{Bibliografia}

BOSQUE PÉREZ, Ramón; COLÓN MORERA, José. Las Carpetas. Persecución política y derechos civiles en Puerto Rico. Río Piedras: CIPDC, 1997.

CONSTITUCIÓN DEL ESTADO LIBRE Y ASOCIADO DE PUERTO RICO, 1952.

GONZÁLEZ CRUZ, Michael. Nacionalismo revolucionario puertorriqueño. La lucha armada, intelectuales y prisiones politicos y de guerra. San Juan: Isla Negra, 2006.

MALDONADO DENIS, Manuel. Puerto Rico. Una interpretación histórico - social. México: Siglo XXI, 1970.

MOSCOSO, Francisco. La Revolución Puertorriqueña de 1868: el Grito de Lares. San Juan: Instituto de Cultura Puertorriqueña, 2003.

PARALITICI, José. La represión contra el independentismo puertorriqueño: 1960-2010. San Juan: Ediciones Gaviota, 2011. 